\title{
Psychosomatic medicine - viability of a discipline
}

\author{
Monika Graninger
}

Since ancient times, the self-evident interrelationship between functions of the brain and the development and regulation of other bodily functions has been used by medical caretakers in many cultures. With the Age of Enlightenment, the use of rational thinking was accompanied by the flourishing of natural science. Still, the biological mechanisms behind many brain functions remain to be decrypted. Modern psychology gallops side by side with the biological deciphering of the hitherto mystical mechanisms of emotions, thoughts, and attitudes. To the modern physician it is becoming clear that there is a bidirectional junction between the brain and the immune system and even the gut bacteria, as is illustrated in this issue.

In practical clinical medicine, any principle is welcome that is capable of reducing the burden of suffering, be the cause biological, psychological, or behavioral. Empirical individual evidence of effectivity together with historically purported beliefs and attitudes gave rise to innumerable methods and teachings of healing. Natural science and biology rightfully claim to provide models and products of unprecedented effectivity (such as vaccinations, surgery, or pharmaceutical merchandise with controlled, safe, and reproducible properties). Recently, many therapeutic disciplines dealing with cognitive-emotional procedures claim to follow "quality criteria." However, still many models of "healing" inherently transcend rational approaches. For an effective solution to the task of "min- imization of health-related suffering," the concept of a liaison between biology-oriented medicine and psychotherapy developed in the last century and it was finally given the name "psychosomatic medicine," as described so elegantly by Deter et al. in this issue.

The practical application of a harmonized and intertwined mélange of methods (albeit sometimes almost ridiculed, like in the word "holistic") is shown by two articles in this special issue, illustrating gastroenterological and cardiological examples.

Far ahead of the times, after the biological-mechanistic decryption of all brain functions, the inclusion of standardized catalogues of psychological and psychotherapeutic skills into the curricula of medical training will make the self-evident concepts of psychosomatic medicine a matter of historical studies.

But today, we still have to assign the task of psychologically oriented medical help to medical specialists and to specially trained general health- care providers. In order to optimize the medical service concept in the German-speaking regions, we have to emphasize the biopsychosocial model.

With this issue of the Wiener Medizinische Wochenschrift, I hope to have illustrated the need for implementing the practical concepts of "psychosomatic medicine."

Conflict of interest M. Graninger declares that she has no competing interests. 